\title{
Nonlinear Vibrations and Chaos in Rectangular Functionally Graded Plates with Thermo-mechanical Coupling
}

\author{
Seyedeh Elnaz Naghibi \\ School of Engineering and Materials Science, Queen Mary, University of London, London, UK
}

\author{
Mojtaba Mahzoon \\ School of Mechanical Engineering, Shiraz University, Shiraz, Iran
}

(Received 19 August 2014; accepted 19 April 2016)

We analyze the nonlinear dynamics of a simply supported, rectangular, and functionally graded plate in terms of a newly derived coupled system of thermo-elasticity and energy equations, which is then expanded here in derivations and explored for chaotic responses through a parameter study in the state space. ${ }^{1}$ The plate properties vary linearly in thickness. Three-dimensional stress-strain relations are considered in general case and nonlinear strain-displacement relations are deployed to account for the plate's large deflection. A lateral harmonic force is applied on the plate, and there is a heat generation source within it and the surfaces are exposed to free convection. By integrating over the thickness, four new thermal parameters are introduced, which together with the midplane displacements constitute a system of seven partial differential equations. These equations are changed into ordinary differential equations in time using Galerkin's approximation and solved by using the $4^{\text {th }}$ order RungeKutta method. Finally, a parameter study is performed and the appropriate conditions resulting in chaotic solutions are determined by using numerical features such as the Lyapunov exponent and power spectrum.

\section{NOMENCLATURE}

$\begin{array}{ll}\bar{u}, \bar{v}, \bar{w} & \begin{array}{l}\text { Particles displacements in } x, y, z \text { direc- } \\ \text { tions }\end{array} \\ u, v, w & \begin{array}{l}\text { Midplane displacements in } x, y, z \text { direc- } \\ \text { tions }\end{array} \\ A, B, C & \text { Dimensionless amplitudes of } u, v, w \\ V & \text { Dimensionless amplitude of } \dot{w} \\ \varepsilon_{i j} & \text { Strain components } \\ \sigma_{i j} & \text { Stress components } \\ \theta & \text { Point-wise plate temperature } \\ \theta_{0} & \text { Initial plate temperature } \\ T & \text { Dimensionless amplitude of } \theta \\ N_{\theta}, M_{\theta}, P_{\theta}, Q_{\theta} & \text { Temperature } 0^{\text {th }} \text { to } 3^{\text {th }} \text { moments } \\ D, E, F, G & \text { Dimensionless amplitudes of } N, M, P, \\ E & \text { and } Q \\ \nu & \text { Modulus of elasticity } \\ \alpha & \text { Poisson's ratio } \\ \rho & \text { Thermal expansion coefficient } \\ k & \text { Density } \\ c & \text { Thermal diffusivity } \\ r & \text { Specific heat capacity } \\ \lambda & \text { Heat generation rate per unit mass } \\ h & \text { Plate side length to thickness ratio } \\ a, b & \text { Plate thickness } \\ \omega & \text { Plate side lengths in } x \text { and } y \text { directions } \\ q_{0} & \text { Frequency of the external force } \\ & \text { Heat transfer coefficient } \\ \text { Amplitude of the external force }\end{array}$

\section{INTRODUCTION}

Functionally graded materials (FGMs) are widely used in aeronautic structures, where they are simultaneously subjected to mechanical and thermal loadings. Thus, presenting a model that combines the mechanical and thermal characteristics increase our understanding of their behavior. In this paper, the coupled problem of thermo-elasticity for the nonlinear dynamics of FGM plates was studied, the governing equations were derived, and after achieving the solution, some parameter values for which trajectories show chaotic behavior were determined.

In the field of linear thermo-elasticity Nowacki has made several fundamental contributions, among which was the derivation of the equations of thermo-elastic vibrations of plates in the coupled case. ${ }^{2}$ He solved the problem of transverse vibrations when the temperature field varied harmonically with time. Kawamura et al. derived the governing equations for thermally induced vibrations of an FGM plate exposed to sinusoidally varying surface temperature. ${ }^{3}$ Xiang and Melnik presented a numerical approach for the general thermo-mechanical problems, which was based on the reduction of the original system of partial differential equations to a system of differential algebraic equations. ${ }^{4}$ They tested the method for a two-dimensional, thermo-elasticity problem. Yang and Shen performed an analysis on free and forced vibrations of initially stressed FGM plates with temperature dependent material properties. ${ }^{5}$ They also studied partially distributed impulsive loads on FGM plates resting on elastic foundations. ${ }^{6}$ Gupta investigated non-linear thickness variation on the thermally-induced vibration of a rectangular plate using a spline technique. ${ }^{7}$ 
A number of papers have been dedicated to the nonlinear free vibrations of plates. Prabhakara and Chia calculated nonlinear frequencies for free flexural large amplitude vibrations of orthotropic rectangular plates with different boundary conditions and showed that for large values of amplitude, the coupling effect of vibrating modes on the nonlinear frequency of the fundamental mode is significant for orthotropic plates. ${ }^{8} \mathrm{Al}-$ lahverdizadeh et al. analyzed free and forced axisymmetric vibrations of a thin circular FGM plate in a thermal environment and determined the stresses and nonlinear natural frequencies using a semi-analytical approach. ${ }^{9}, 10$ Chang and Chian presented an analytical study on free vibrations of a heated orthotropic rectangular thin plate under various boundary conditions. ${ }^{11}$ Xuefeng et al. studied nonlinear thermo-elastic free vibrations of circular plates that had simply supported and clamped boundary conditions. ${ }^{12,13}$

Furthermore, Praveen and Reddy analyzed the static and dynamic response of FGM plates using a plate finite element that accounts for the transverse shear strains, rotary inertia, and moderately large rotations in von Karman sense. ${ }^{14}$ Huang and Shen investigated nonlinear vibrations of an FGM plate in thermal environments, accounting for heat conduction and temperature dependent material. ${ }^{15}$ Woo and Meguid provided an analytical solution for the coupled large deflection of plates and shallow shells made of functionally graded material. They made use of two-dimensional stress-strain relations and studied the effect of thermo-mechanical coupling on the FGM shell response. ${ }^{16}$ In another survey, Woo et al. discussed the effect of material properties, boundary conditions, and thermal loading on nonlinear dynamics of a freely vibrating FGM plate. ${ }^{17}$ Chang and Wan presented an analytical method to investigate large amplitude thermo-mechanically coupled vibrations of rectangular elastic thin plates with various boundary conditions. ${ }^{18}$ Hao et al. studied the nonlinear dynamics of a simply supported rectangular FGM plate subjected to transverse and in-plane excitations in a time dependent thermal environment. ${ }^{19}$ Yeh analyzed large amplitude thermo-mechanically coupled vibrations of simply supported orthotropic rectangular thin plates. ${ }^{20} \mathrm{He}$ discussed the effect of changing different parameters on thermal damping of the plate. Alijani and Amabili investigated non-linear parametric instability of rectangular FGM plates in thermal environments using a multi-degreeof-freedom energy approach. ${ }^{21}$

Among works done on the chaotic vibrations of plates, we can mention the derivation of the nonlinear dynamic equation of a harmonically forced elliptic plate in the presence of a thermal field by Qiang et al. ${ }^{22}$ They used the Melnikov function method to give the critical condition for chaotic motion and to discuss the path to chaos. Ribeiro performed an analysis on geometrically nonlinear vibrations of linear elastic and isotropic plates under the combined effect of thermal fields and mechanical excitations and studied transitions from periodic to nonperiodic motions. ${ }^{23}$ Both him and Duarte also investigated the effect of fibers' angle in nonlinear thermo-elastic vibrations of composite laminated plates. ${ }^{24}$ Lai et al. determined the conditions leading to chaotic motion for large deflections of a simply supported rectangular plate by examining the fractal dimension and the maximum the Lyapunov exponent. ${ }^{25}$ They used the same approach to detect chaos in large deflections of rectangular plates with thermo-mechanical coupling. ${ }^{26}$ In another investigation, Yeh and Chen found the conditions that re- sulted in chaos for a simply supported circular plate of thermomechanical coupling with variable thickness. ${ }^{27}$ Zhang et al. analyzed the nonlinear dynamics and chaos of a simply supported rectangular orthotropic FGM plate in a thermal environment in which heat conduction and temperature-dependent material properties were taken into account. ${ }^{28}$ Hao et al. observed chaos in nonlinear dynamics of a rectangular FGM plate in a thermal environment. ${ }^{29}$

However, none of the referred papers have considered the thermo-mechanical coupling for nonlinear forced vibrations of functionally graded plates. The novelty of this paper is the full derivation of the governing equations in the threedimensional case while accounting for geometrical nonlinearity, non-homogeneity, coupled fields of temperature, and displacement in the extension of our preliminary research, which only includes the general framework for this purpose. ${ }^{1}$ Additionally, we have performed a parameter study in the state space to find the parameter values that trigger chaotic solutions. In the following sections, the equations of motion and energy equation are first derived for a nonlinear thermo-elastic FGM plate and then a solution strategy is proposed for solving them. Section three is devoted to a parameter study and discusses the behavior of the resulting responses, with a focus on detecting chaos. Finally, the conclusions are drawn in the last section.

\section{METHOD}

The first aim of this paper is to derive the equations of motion and the energy equation considering thermo-mechanical coupling for a rectangular plate made of functionally graded material. Equations of motion were derived on the basis of displacement formulation. ${ }^{30}$ Lagrangian finite strain tensor is defined as: ${ }^{31}$

$$
\varepsilon_{i j}=\frac{1}{2}\left(\frac{\partial u_{i}}{\partial x_{j}}+\frac{\partial u_{j}}{\partial x_{i}}\right)+\frac{1}{2} \frac{\partial u_{m}}{\partial x_{i}} \frac{\partial u_{m}}{\partial x_{j}} .
$$

Based on Kirchhoff hypothesis, the displacements of various points in the plate thickness are linearly related to those of the mid-plane as: $:^{32}$

$$
\begin{aligned}
\vec{u}(x, y, z, t) & =u(x, y, t)-z \frac{\partial w}{\partial x} \\
\vec{v}(x, y, z, t) & =v(x, y, t)-z \frac{\partial w}{\partial y} \\
\vec{w}(x, y, z, t) & =w(x, y, t) .
\end{aligned}
$$

Substituting Eq. (2) in Eq. (1), strain components in terms of mid-plane displacements were found. ${ }^{33}$ Additionally, generalized thermo-elastic constitutive law for isotropic material was given by: ${ }^{30}$

$$
\sigma_{i j}=\lambda \varepsilon_{k k} \delta_{i j}+2 \mu \varepsilon_{i j}-(3 \lambda+2 \mu) \alpha\left(\theta-\theta_{0}\right) ;
$$

where,

$$
\begin{aligned}
& \lambda=\frac{E \nu}{(1+\nu)(1-2 \nu)} ; \\
& \mu=\frac{E}{2(1+\nu)} .
\end{aligned}
$$

By using Eq. (3), stress tensor components were found in terms of mid-plane displacements as: 


$$
\begin{aligned}
& \sigma_{x x}=\frac{E \nu}{(1+\nu)(1-2 \nu)}\left[\frac{\partial u}{\partial x}+\frac{\partial v}{\partial y}+\left(\frac{\partial w}{\partial x}\right)^{2}+\left(\frac{\partial w}{\partial y}\right)^{2}-\right. \\
& \left.z \frac{\partial^{2} w}{\partial x^{2}}-z \frac{\partial^{2} w}{\partial y^{2}}\right]+\frac{E}{(1+\nu)}\left[\frac{\partial u}{\partial x}+\frac{1}{2}\left(\frac{\partial w}{\partial x}\right)^{2}-\right. \\
& \left.z \frac{\partial^{2} w}{\partial x^{2}}\right]-\frac{E \alpha}{(1-2 \nu)}\left(\theta-\theta_{0}\right) \text {; } \\
& \sigma_{y y}=\frac{E \nu}{(1+\nu)(1-2 \nu)}\left[\frac{\partial u}{\partial x}+\frac{\partial v}{\partial y}+\left(\frac{\partial w}{\partial x}\right)^{2}+\left(\frac{\partial w}{\partial y}\right)^{2}-\right. \\
& \left.z \frac{\partial^{2} w}{\partial x^{2}}-z \frac{\partial^{2} w}{\partial y^{2}}\right]+\frac{E}{(1+\nu)}\left[\frac{\partial v}{\partial y}+\frac{1}{2}\left(\frac{\partial w}{\partial y}\right)^{2}-\right. \\
& \left.z \frac{\partial^{2} w}{\partial y^{2}}\right]-\frac{E \alpha}{(1-2 \nu)}\left(\theta-\theta_{0}\right) \text {; } \\
& \sigma_{z z}=\frac{E \nu}{(1+\nu)(1-2 \nu)}\left[\frac{\partial u}{\partial x}+\frac{\partial v}{\partial y}+\left(\frac{\partial w}{\partial x}\right)^{2}+\left(\frac{\partial w}{\partial y}\right)^{2}-\right. \\
& \left.z \frac{\partial^{2} w}{\partial x^{2}}-z \frac{\partial^{2} w}{\partial y^{2}}\right]+\frac{E}{(1+\nu)}\left[\frac{1}{2}\left(\frac{\partial w}{\partial x}\right)^{2}+\right. \\
& \left.\frac{1}{2}\left(\frac{\partial w}{\partial y}\right)^{2}\right]-\frac{E \alpha}{(1-2 \nu)}\left(\theta-\theta_{0}\right) \text {; } \\
& \sigma_{x y}=\frac{E}{(1+\nu)}\left[\frac{1}{2}\left(\frac{\partial u}{\partial y}+\frac{\partial v}{\partial x}+\frac{\partial w}{\partial x} \frac{\partial w}{\partial y}\right)-z \frac{\partial^{2} w}{\partial x \partial y}\right] \\
& \sigma_{x z}=\frac{E}{(1+\nu)}\left[\frac{1}{2} z \frac{\partial^{2} w}{\partial x^{2}} \frac{\partial w}{\partial x}+\frac{1}{2} z \frac{\partial^{2} w}{\partial x \partial y} \frac{\partial w}{\partial y}-\frac{1}{2} \frac{\partial u}{\partial x} \frac{\partial w}{\partial x}-\right. \\
& \left.\frac{1}{2} \frac{\partial v}{\partial x} \frac{\partial w}{\partial y}\right] \\
& \sigma_{y z}=\frac{E}{(1+\nu)}\left[\frac{1}{2} z \frac{\partial^{2} w}{\partial y^{2}} \frac{\partial w}{\partial y}+\frac{1}{2} z \frac{\partial^{2} w}{\partial x \partial y} \frac{\partial w}{\partial x}-\frac{1}{2} \frac{\partial u}{\partial y} \frac{\partial w}{\partial x}-\right. \\
& \left.\frac{1}{2} \frac{\partial v}{\partial y} \frac{\partial w}{\partial y}\right]
\end{aligned}
$$

It was assumed that the volume fractions, $x_{A}$ and $x_{B}$, and consequently all extensive properties, varied linearly in thickness. Thus, a plate typical property $P$ was expressed as:

$$
\begin{aligned}
P & =x_{A} P_{A}+x_{B} P_{B}=\frac{P_{A}+P_{B}}{2}+\frac{z\left(P_{B}-P_{A}\right)}{h} \\
& =P_{\text {avg }}+\frac{z}{h} \Delta P ;
\end{aligned}
$$

where $A$ and $B$ are the two types of material used at the lower and upper surfaces respectively.

The equations of motion in the absence of body forces had the form:

$$
\sigma_{i j, j}=\rho \ddot{\bar{u}}_{i} .
$$

In order to reduce spatial dimensions of Eq. (13), we integrated both sides of each equation over the thickness. In this way, we let the property variations emerge in the equations of motion. This integration yielded:

$$
\frac{\partial N_{x x}}{\partial x}+\frac{\partial N_{x y}}{\partial y}+\left.\sigma_{x z}\right|_{-h / 2} ^{h / 2}=\rho_{\text {avg }} h \frac{\partial^{2} u}{\partial t^{2}}-\frac{h^{2} \Delta \rho}{12} \frac{\partial^{3} w}{\partial x \partial t^{2}} ;
$$

$$
\begin{gathered}
\frac{\partial N_{x y}}{\partial x}+\frac{\partial N_{y y}}{\partial y}+\left.\sigma_{y z}\right|_{-h / 2} ^{h / 2}=\rho_{\text {avg }} h \frac{\partial^{2} v}{\partial t^{2}}-\frac{h^{2} \Delta \rho}{12} \frac{\partial^{3} w}{\partial y \partial t^{2}} \\
\frac{\partial Q_{x}}{\partial x}+\frac{\partial Q_{y}}{\partial y}+\left.\sigma_{z z}\right|_{-h / 2} ^{h / 2}=\rho_{\text {avg }} h \frac{\partial^{2} w}{\partial t^{2}}
\end{gathered}
$$

where,

$$
\begin{aligned}
N_{x x} & =\int_{-h / 2}^{h / 2} \sigma_{x x} d z ; & N_{y y} & =\int_{-h / 2}^{h / 2} \sigma_{y y} d z ; \\
N_{x y} & =\int_{-h / 2}^{h / 2} \sigma_{x y} d z ; & Q_{x} & =\int_{-h / 2}^{h / 2} \sigma_{x z} d z ; \\
Q_{y} & =\int_{-h / 2}^{h / 2} \sigma_{y z} d z ; & \left.\sigma_{z z}\right|_{-h / 2} ^{h / 2} & =q_{0} \sin (\omega t) ;
\end{aligned}
$$

and $\left.\sigma_{x z}\right|_{-h / 2} ^{h / 2}$ and $\left.\sigma_{y z}\right|_{-h / 2} ^{h / 2}$ can be found from Eqs. (10) and (11). $N_{x x}, N_{y y}$, and $N_{x y}$ were obtained by directly integrating Eqs. (6), (7), and (9). Thus, the equations of motion in the $x$ and $y$ directions were given by:

$$
\begin{gathered}
\frac{E_{\text {avg }} h(1-\nu)}{(1+\nu)(1-2 \nu)} \frac{\partial^{2} u}{\partial x^{2}}+\frac{E_{\text {avg }} h}{2(1+\nu)(1-2 \nu)} \frac{\partial^{2} v}{\partial x \partial y}+ \\
\frac{E_{\text {avg }} h}{2(1+\nu)} \frac{\partial^{2} u}{\partial y^{2}}+\frac{E_{\text {avg } h(3-2 \nu)}(1+\nu)(1-2 \nu)}{2(x)} \frac{\partial w}{\partial x^{2}}+ \\
\frac{E_{\text {avg }} h}{(1+\nu)(1-2 \nu)} \frac{\partial w}{\partial y} \frac{\partial^{2} w}{\partial x \partial y}-\frac{\Delta E h^{2}(1-\nu)}{12(1+\nu)(1-2 \nu)} \\
\left(\frac{\partial^{3} w}{\partial x^{3}}+\frac{\partial^{3} w}{\partial x \partial y^{2}}\right)+\frac{E_{\text {avg }} h}{2(1+\nu)} \frac{\partial w}{\partial x} \frac{\partial^{2} w}{\partial y^{2}}- \\
\frac{1}{1-2 \nu}\left[E_{\text {avg }} \alpha_{\text {avg }} \frac{\partial}{\partial x} \int_{-h / 2}^{h / 2}\left(\theta-\theta_{0}\right) d z+\right. \\
\frac{\left(E_{\text {avg }} \Delta \alpha+\alpha_{\text {avg }} \Delta E\right)}{h} \frac{\partial}{\partial x} \int_{-h / 2}^{h / 2} z\left(\theta-\theta_{0}\right) d z+ \\
\left.\frac{\Delta E \Delta \alpha}{h^{2}} \frac{\partial}{\partial x} \int_{-h / 2}^{h / 2} z^{2}\left(\theta-\theta_{0}\right) d z\right]= \\
\rho_{\text {avg }} h \frac{\partial^{2} u}{\partial t^{2}}-\frac{h^{2} \Delta \rho}{12} \frac{\partial^{3} w}{\partial x \partial t^{2}}
\end{gathered}
$$

$$
\begin{gathered}
\frac{E_{\text {avg }} h(1-\nu)}{(1+\nu)(1-2 \nu)} \frac{\partial^{2} v}{\partial y^{2}}+\frac{E_{\text {avg }} h}{2(1+\nu)(1-2 \nu)} \frac{\partial^{2} u}{\partial x \partial y}+ \\
\frac{E_{\text {avg }} h}{2(1+\nu)} \frac{\partial^{2} v}{\partial x^{2}}+\frac{E_{\text {avg }} h(3-2 \nu)}{2(1+\nu)(1-2 \nu)} \frac{\partial w}{\partial y} \frac{\partial^{2} w}{\partial y^{2}}+ \\
\frac{E_{\text {avg }} h}{(1+\nu)(1-2 \nu)} \frac{\partial w}{\partial x} \frac{\partial^{2} w}{\partial x \partial y}-\frac{\Delta E h^{2}(1-\nu)}{12(1+\nu)(1-2 \nu)} \\
\left(\frac{\partial^{3} w}{\partial y^{3}}+\frac{\partial^{3} w}{\partial y \partial x^{2}}\right)+\frac{E_{\text {avg }} h}{2(1+\nu)} \frac{\partial w}{\partial y} \frac{\left(\partial^{2} w\right.}{\partial x^{2}}- \\
\frac{1}{1-2 \nu}\left[E_{\text {avg }} \alpha_{\text {avg }} \frac{\partial}{\partial y} \int_{-h / 2}^{h / 2}\left(\theta-\theta_{0}\right) d z+\right. \\
\frac{\left(E_{\text {avg }} \Delta \alpha+\alpha_{\text {avg }} \Delta E\right)}{h} \frac{\partial}{\partial y} \int_{-h / 2}^{h / 2} z\left(\theta-\theta_{0}\right) d z+ \\
\left.\frac{\Delta E \Delta \alpha}{h^{2}} \frac{\partial}{\partial y} \int_{-h / 2}^{h / 2} z^{2}\left(\theta-\theta_{0}\right) d z\right]= \\
\rho_{\text {avg }} h \frac{\partial^{2} v}{\partial t^{2}}-\frac{h^{2} \Delta \rho}{12} \frac{\partial^{3} w}{\partial y \partial t^{2}} .
\end{gathered}
$$


However, in order to derive the equation of motion in the $z$ direction, $Q_{x}$ and $Q_{y}$ were obtained by multiplying the equations of motion in the $x$ and $y$ direction by $z$ and integrating them over the thickness. This yielded:

$$
\begin{aligned}
\frac{\partial M_{x x}}{\partial x}+\frac{\partial M_{x y}}{\partial y} & -Q_{x}+\left.\left(z \sigma_{x z}\right)\right|_{-h / 2} ^{h / 2}= \\
& -\frac{h^{3}}{12} \rho_{\text {avg }} \frac{\partial^{3} w}{\partial t^{2} \partial x}+\frac{h^{2}}{12} \Delta \rho \frac{\partial^{2} u}{\partial t^{2}} ; \\
\frac{\partial M_{x y}}{\partial x}+\frac{\partial M_{y y}}{\partial y} & -Q_{y}+\left.\left(z \sigma_{y z}\right)\right|_{-h / 2} ^{h / 2}= \\
& -\frac{h^{3}}{12} \rho_{\text {avg }} \frac{\partial^{3} w}{\partial t^{2} \partial y}+\frac{h^{2}}{12} \Delta \rho \frac{\partial^{2} u}{\partial t^{2}} ;
\end{aligned}
$$

where,

$$
\begin{gathered}
M_{x x}=\int_{-h / 2}^{h / 2} z \sigma_{x x} d z ; \quad M_{y y}=\int_{-h / 2}^{h / 2} z \sigma_{y y} d z ; \\
M_{x y}=\int_{-h / 2}^{h / 2} z \sigma_{x y} d z
\end{gathered}
$$

and $\left.\left(z \sigma_{x z}\right)\right|_{-h / 2} ^{h / 2}$ and $\left.\left(z \sigma_{y z}\right)\right|_{-h / 2} ^{h / 2}$ could be calculated from Eqs. (10) and (11). Differentiating Eq. (20) with respect to $x$ and Eq. (21) with respect to $y$ and substituting the resulting $\frac{\partial Q_{x}}{\partial x}$ and $\frac{\partial Q_{y}}{\partial y}$ together with the stress components in Eq. (16), resulted in the equation of motion in the $z$ direction, which was expressed as:

$$
\begin{aligned}
& \frac{h^{2} \Delta E(1-\nu)}{12(1+\nu)(1-2 \nu)}\left[\frac{\partial^{3} u}{\partial y^{2} \partial x}+\frac{\partial^{3} v}{\partial x^{2} \partial y}+\frac{\partial^{3} u}{\partial x^{3}}+\frac{\partial^{3} v}{\partial y^{3}}\right]+ \\
& \frac{(2-3 \nu) h^{2} \Delta E}{6(1+\nu)(1-2 \nu)}\left[\left(\frac{\partial^{2} w}{\partial x^{2}}\right)^{2}+\left(\frac{\partial^{2} w}{\partial y^{2}}\right)^{2}+\frac{\partial w}{\partial x} \frac{\partial^{3} w}{\partial x^{3}}+\right. \\
& \left.\frac{\partial w}{\partial y} \frac{\partial^{3} w}{\partial y^{3}}+\frac{\partial w}{\partial x} \frac{\partial^{3} w}{\partial y^{2} \partial x}+\frac{\partial w}{\partial y} \frac{\partial^{3} w}{\partial x^{2} \partial y}\right]+ \\
& \frac{h^{2} \Delta E(7-10 \nu)}{12(1+\nu)(1-2 \nu)}\left(\frac{\partial^{2} w}{\partial x \partial y}\right)^{2}+\frac{h^{2} \Delta E}{12(1+\nu)} \frac{\partial^{2} w}{\partial x^{2}} \frac{\partial^{2} w}{\partial y^{2}}- \\
& \frac{E_{\mathrm{avg}} h^{3}(1-\nu)}{12(1+\nu)(1-2 \nu)} \nabla^{4} w-\frac{h E_{\mathrm{avg}}}{(1+\nu)}\left(\frac{\partial^{2} u}{\partial x^{2}} \frac{\partial w}{\partial x}+\right. \\
& \frac{\partial u}{\partial x} \frac{\partial^{2} w}{\partial x^{2}}+\frac{\partial^{2} v}{\partial x^{2}} \frac{\partial w}{\partial y}+\frac{\partial v}{\partial x} \frac{\partial^{2} w}{\partial x \partial y}+\frac{\partial^{2} u}{\partial y^{2}} \frac{\partial w}{\partial x}+ \\
& \left.\frac{\partial u}{\partial y} \frac{\partial^{2} w}{\partial x \partial y}+\frac{\partial v}{\partial y} \frac{\partial^{2} w}{\partial y^{2}}+\frac{\partial^{2} v}{\partial y^{2}} \frac{\partial w}{\partial y}\right)+ \\
& \frac{h^{3}}{12} \rho_{\text {avg }} \frac{\partial^{4} w}{\partial t^{2} \partial x^{2}}-\frac{h^{2}}{12} \Delta \rho \frac{\partial^{3} u}{\partial x \partial t^{2}}+\frac{h^{3}}{12} \rho_{\text {avg }} \frac{\partial^{4} w}{\partial t^{2} \partial y^{2}}- \\
& \frac{h^{2}}{12} \Delta \rho \frac{\partial^{3} v}{\partial y \partial t^{2}}-\frac{1}{1-2 \nu}\left[E_{\text {avg }} \alpha_{\text {avg }} \nabla^{2} \int_{-h / 2}^{h / 2} z\left(\theta-\theta_{0}\right) d z+\right. \\
& \frac{\left(E_{\mathrm{avg}} \Delta \alpha+\alpha_{\mathrm{avg}} \Delta E\right)}{h} \nabla^{2} \int_{-h / 2}^{h / 2} z^{2}\left(\theta-\theta_{0}\right) d z+ \\
& \left.\frac{\Delta E \Delta \alpha}{h^{2}} \nabla^{2} \int_{-h / 2}^{h / 2} z^{3}\left(\theta-\theta_{0}\right) d z\right]+q_{0} \sin (\omega t)= \\
& \rho_{\text {avg }} h \frac{\partial^{2} w}{\partial t^{2}} \text {. }
\end{aligned}
$$

As a result of reducing spatial dimensions of the equations of motion, these equations were changed into integro- differential equations. By introducing the four following parameters, the equations could remain in differential form:

$$
\begin{array}{rlrl}
N_{\theta} & \equiv \int_{-h / 2}^{h / 2}\left(\theta-\theta_{0}\right) d z ; & M_{\theta} \equiv \int_{-h / 2}^{h / 2} z\left(\theta-\theta_{0}\right) d z ; \\
P_{\theta} \equiv \int_{-h / 2}^{h / 2} z^{2}\left(\theta-\theta_{0}\right) d z ; & Q_{\theta} \equiv \int_{-h / 2}^{h / 2} z^{3}\left(\theta-\theta_{0}\right) d z .
\end{array}
$$

To specify these four parameters together with displacement components in the $x, y$, and $z$ directions, seven independent equations were needed. So far, three equations were derived, and the other four were deduced from the energy equation.

The energy equation for a thermo-elastic continuous media experiencing small temperature changed in the presence of heat generation: ${ }^{34}$

$$
\rho r+\nabla(k \nabla \theta)-\rho c \dot{\theta}-\beta \theta_{o} \dot{\varepsilon}_{m m}=0 ;
$$

where,

$$
\beta=3 k \alpha=\frac{E \alpha}{(1-2 \nu)} .
$$

Equation (25) was also integrated over the thickness and solved together with thermo-elasticity equations. However, since we introduced four new thermal variables in Eq. (24), and because we were interested in their dynamics, we still needed three other equations for the closure of the problem. Thus, in addition to mere integration, we multiplied Eq. (25) by $z, z^{2}$, and $z^{3}$ and integrated over the thickness. As a result, by using Eqs. (1), (12), (24), and (25) and finite integration rules, the four following equations were added to the equations of motion:

$$
\begin{aligned}
& \rho_{\mathrm{avg}} h r+k_{\mathrm{avg}} \nabla^{2} N_{\theta}+\frac{\Delta k}{h} \nabla^{2} M_{\theta}+\left.k_{\mathrm{avg}} \frac{\partial \theta}{\partial z}\right|_{-h / 2} ^{h / 2}+ \\
& \left.\frac{\Delta k}{h} z \frac{\partial \theta}{\partial z}\right|_{-h / 2} ^{h / 2}-\rho_{\mathrm{avg}} c_{\mathrm{avg}} \frac{\partial N_{\theta}}{\partial t}-\frac{\left(\rho_{\mathrm{avg}} \Delta c+c_{\mathrm{avg}} \Delta \rho\right)}{h} \frac{\partial M_{\theta}}{\partial t}- \\
& \frac{\Delta c \Delta \rho}{h^{2}} \frac{\partial P_{\theta}}{\partial t}-\frac{\theta_{0} h}{(1-2 \nu)}\left(E_{\mathrm{avg}} \alpha_{\mathrm{avg}}+\frac{\Delta E \delta \alpha}{12}\right) \cdot \\
& \left(\frac{\partial^{2} u}{\partial t \partial x}+\frac{\partial^{2} v}{\partial t \partial y}+2 \frac{\partial w}{\partial x} \frac{\partial^{2} w}{\partial t \partial x}+2 \frac{\partial w}{\partial y} \frac{\partial^{2} w}{\partial t \partial y}\right)+ \\
& \frac{\theta_{0} h^{2}}{12(1-2 \nu)}\left(E_{\mathrm{avg}} \Delta \alpha+\alpha_{\mathrm{avg}} \Delta E\right)\left(\frac{\partial^{3} w}{\partial t \partial x^{2}}+\frac{\partial^{3} w}{\partial t \partial y^{2}}\right)=0
\end{aligned}
$$

$$
\begin{aligned}
& \frac{h^{2} \Delta \rho r}{12}+k_{\mathrm{avg}} \nabla^{2} M_{\theta}+\frac{\Delta k}{h} \nabla^{2} P_{\theta}+\left.k_{\mathrm{avg}} z \frac{\partial \theta}{\partial z}\right|_{-h / 2} ^{h / 2}- \\
& \left.k_{\mathrm{avg}} \theta\right|_{-h / 2} ^{h / 2}+\frac{\Delta k}{h}\left(\left.z^{2} \frac{\partial \theta}{\partial z}\right|_{-h / 2} ^{h / 2}-\left.z \theta\right|_{-h / 2} ^{h / 2}+h \theta_{0}+N_{\theta}\right)- \\
& \rho_{\mathrm{avg}} c_{\mathrm{avg}} \frac{\partial M_{\theta}}{\partial t}-\frac{\left(\rho_{\mathrm{avg}} \Delta c+c_{\mathrm{avg}} \Delta \rho\right)}{h} \frac{\partial P_{\theta}}{\partial t}-\frac{\Delta c \Delta \rho}{h^{2}} \frac{\partial Q_{\theta}}{\partial t}- \\
& \frac{\theta_{0} h^{2}}{12(1-2 \nu)}\left(E_{\mathrm{avg}} \Delta \alpha+\alpha_{\mathrm{avg}} \Delta E\right) \cdot \\
& \left(\frac{\partial^{2} u}{\partial t \partial x}+\frac{\partial^{2} v}{\partial t \partial y}+2 \frac{\partial w}{\partial x} \frac{\partial^{2} w}{\partial t \partial x}+2 \frac{\partial w}{\partial y} \frac{\partial^{2} w}{\partial t \partial y}\right)+ \\
& \frac{\theta_{0} h^{3}}{(1-2 \nu)}\left(\frac{E_{\mathrm{avg}} \alpha_{\mathrm{avg}}}{12}+\frac{\Delta E \Delta \alpha}{80}\right)\left(\frac{\partial^{3} w}{\partial t \partial x^{2}}+\frac{\partial^{3} w}{\partial t \partial y^{2}}\right)=0
\end{aligned}
$$




$$
\begin{aligned}
& \frac{h^{3} \rho_{\text {avg }} r}{12}+k_{\text {avg }} \nabla^{2} P_{\theta}+\frac{\Delta k}{h} \nabla^{2} Q_{\theta}+ \\
& k_{\text {avg }}\left(\left.z^{2} \frac{\partial \theta}{\partial z}\right|_{-h / 2} ^{h / 2}-\left.2 z \theta\right|_{-h / 2} ^{h / 2}+2 h \theta_{0}+2 N_{\theta}\right)+ \\
& \frac{\Delta k}{h}\left(\left.z^{3} \frac{\partial \theta}{\partial z}\right|_{-h / 2} ^{h / 2}-\left.2 z^{2} \theta\right|_{-h / 2} ^{h / 2}+4 M_{\theta}\right)-\rho_{\text {avg }} c_{\text {avg }} \frac{\partial P_{\theta}}{\partial t}- \\
& \frac{\left(\rho_{\text {avg }} \Delta c+c_{\text {avg }} \Delta \rho\right)}{h} \frac{\partial Q_{\theta}}{\partial t}-\frac{\Delta c \Delta \rho}{h^{2}} \frac{\partial}{\partial t} \int_{-h / 2}^{h / 2} z^{4} \theta d z- \\
& \frac{\theta_{0} h^{3}}{(1-2 \nu)}\left(\frac{E_{\text {avg }} \alpha_{\text {avg }}}{12}+\frac{\Delta E \Delta \alpha}{80}\right) . \\
& \left(\frac{\partial^{2} u}{\partial t \partial x}+\frac{\partial^{2} v}{\partial t \partial y}+2 \frac{\partial w}{\partial x} \frac{\partial^{2} w}{\partial t \partial x}+2 \frac{\partial w}{\partial y} \frac{\partial^{2} w}{\partial t \partial y}\right)+ \\
& \frac{\theta_{0} h^{4}}{80(1-2 \nu)}\left(E_{\text {avg }} \Delta \alpha+\alpha_{\text {avg }} \Delta E\right)\left(\frac{\partial^{3} w}{\partial t \partial x^{2}}+\frac{\partial^{3} w}{\partial t \partial y^{2}}\right)=0 ;
\end{aligned}
$$

$$
\begin{aligned}
& \frac{h^{4} \Delta \rho r}{80}+k_{\mathrm{avg}} \nabla^{2} Q_{\theta}+\frac{\Delta k}{h} \nabla^{2} \int_{-h / 2}^{h / 2} z^{4} \theta d z+ \\
& k_{\mathrm{avg}}\left(\left.z^{3} \frac{\partial \theta}{\partial z}\right|_{-h / 2} ^{h / 2}-\left.3 z^{2} \theta\right|_{-h / 2} ^{h / 2}+6 M_{\theta}\right)+ \\
& \frac{\Delta k}{h}\left(\left.z^{4} \frac{\partial \theta}{\partial z}\right|_{-h / 2} ^{h / 2}-\left.3 z^{3} \theta\right|_{-h / 2} ^{h / 2}+9 P_{\theta}+\frac{3}{4} h^{3} \theta_{0}\right)- \\
& \rho_{\mathrm{avg}} c_{\mathrm{avg}} \frac{\partial Q_{\theta}}{\partial t}-\frac{\left(\rho_{\mathrm{avg}} \Delta c+c_{\mathrm{avg}} \Delta \rho\right)}{h} \int_{-h / 2}^{h / 2} z^{4} \theta d z- \\
& \frac{\Delta c \Delta \rho}{h^{2}} \frac{\partial}{\partial t} \int_{-h / 2}^{h / 2} z^{5} \theta d z-\frac{\theta_{0} h^{4}}{80(1-2 \nu)}\left(E_{\mathrm{avg}} \Delta \alpha+\alpha_{\mathrm{avg}} \Delta E\right) . \\
& \left(\frac{\partial^{2} u}{\partial t \partial x}+\frac{\partial^{2} v}{\partial t \partial y}+2 \frac{\partial w}{\partial x} \frac{\partial^{2} w}{\partial t \partial x}+2 \frac{\partial w}{\partial y} \frac{\partial^{2} w}{\partial t \partial y}\right)+ \\
& \frac{\theta_{0} h^{5}}{(1-2 \nu)}\left(\frac{E_{\mathrm{avg}} \alpha_{\mathrm{avg}}}{80}+\frac{\Delta E \Delta \alpha}{448}\right)\left(\frac{\partial^{3} w}{\partial t \partial x^{2}}+\frac{\partial^{3} w}{\partial t \partial y^{2}}\right)=0 ;
\end{aligned}
$$

where,

$$
\nabla^{2} \equiv \frac{\partial^{2}}{\partial x^{2}}+\frac{\partial^{2}}{\partial y^{2}} .
$$

On the basis of Galerkin's single term approximation, the dependent variables' functionality of the independent variables $x, y, z$, and $t$ was assumed as:

$$
\begin{aligned}
u(x, y, t) & =A(t) \sin \left(\frac{2 m \pi x}{a}\right) \sin \left(\frac{2 n \pi y}{b}\right) ; \\
v(x, y, t) & =B(t) \sin \left(\frac{2 m \pi x}{a}\right) \sin \left(\frac{2 n \pi y}{b}\right) ; \\
w(x, y, t) & =C(t) \sin \left(\frac{2 m \pi x}{a}\right) \sin \left(\frac{2 n \pi y}{b}\right) ; \\
N_{\theta}(x, y, t) & =h \theta_{0} D(t) \sin \left(\frac{m \pi x}{a}\right) \sin \left(\frac{n \pi y}{b}\right) ; \\
M_{\theta}(x, y, t) & =h^{2} \theta_{0} E(t) \sin \left(\frac{m \pi x}{a}\right) \sin \left(\frac{n \pi y}{b}\right) ; \\
P_{\theta}(x, y, t) & =h^{3} \theta_{0} F(t) \sin \left(\frac{m \pi x}{a}\right) \sin \left(\frac{n \pi y}{b}\right) ; \\
Q_{\theta}(x, y, t) & =h^{4} \theta_{0} G(t) \sin \left(\frac{m \pi x}{a}\right) \sin \left(\frac{n \pi y}{b}\right) ; \\
\theta(x, y, z, t)-\theta_{0} & =\left(\theta_{\infty}-\theta_{0}\right) T(z, t) \sin \left(\frac{m \pi x}{a}\right) \sin \left(\frac{n \pi y}{b}\right) .
\end{aligned}
$$

It should be noted that the assumed functions satisfied the problem's boundary conditions, which were simply supported conditions for the displacement components and constant temperature edges for the energy equation:

$$
\begin{aligned}
& u=v=w=\frac{\partial^{2} w}{\partial x^{2}}=0 \quad \& \quad \theta=\theta_{0} \quad \text { at } \quad x=0, a \\
& u=v=w=\frac{\partial^{2} w}{\partial y^{2}}=0 \quad \& \quad \theta=\theta_{0} \quad \text { at } \quad y=0, b .
\end{aligned}
$$

After substituting the above functions in the governing equations, Eqs. (18) and (19) were weighted by $\sin \left(\frac{2 m \pi x}{a}\right) \sin \left(\frac{2 n \pi y}{b}\right)$ and Eqs. (23), (27), (28), (29), and (30) by $\sin \left(\frac{m \pi x}{a}\right) \sin \left(\frac{n \pi y}{b}\right)$. Subsequently, the resulting equations were integrated over the plate's surface and a system of seven ordinary differential equations was achieved. These equations were rendered dimensionless by introducing the following parameters:

$$
\begin{array}{lrr}
x=x^{*} a ; \quad y=y^{*} b ; \quad z=z^{*} h ; & t=\frac{2 \pi t^{*}}{\omega} ; \\
\lambda=\frac{a}{h} ; \quad u=u^{*} u_{0} ; \quad v=v_{0} v^{*} ; & w=w^{*} w_{0} ; \\
\theta-\theta_{0}=\left(\theta_{\infty}-\theta_{0}\right) \theta^{*}=\Delta \theta_{0} \theta^{*} ; & N_{\theta}=h \theta_{0} N_{\theta}^{*} ; \\
M_{\theta}=h^{2} \theta_{0} M_{\theta}^{*} ; \quad P_{\theta}=h^{3} \theta_{0} P_{\theta}^{*} ; & Q_{\theta}=h^{4} \theta_{0} Q_{\theta}^{*} ; \\
E_{\text {avg }}=E_{B} E_{\text {avg }}^{*} ; & \alpha_{\text {avg }}=\alpha_{B} \alpha_{\text {avg }}^{*} ; & c_{\text {avg }}=c_{A} c_{\text {avg }}^{*} ; \\
k_{\text {avg }}=k_{B} k_{\text {avg }}^{*} \quad & \rho_{\text {avg }}=\rho_{B} \rho_{\text {avg }}^{*} ; & \\
\Delta E=E_{B} \Delta E^{*} ; & \Delta \alpha=\alpha_{B} \Delta \alpha^{*} ; & \Delta c=c_{A} \Delta c^{*} ; \\
\Delta k=k_{B} \Delta k^{*} ; & \Delta \rho=\rho_{B} \Delta \rho^{*} . &
\end{array}
$$

Each material property was non-dimensionalied, with its maximum value belonging to either material type. Material A was considered to be Aremco Macor machinable glass ceramic and material B was considered to be AISI 1010 steel. The values of the properties are given in Table 1 for both material types. ${ }^{35}$

By letting $m=n=1$ and $a=b$, the governing equations for a square plate using a single term Galerkin's approximation was eventually derived as:

$$
\begin{gathered}
\frac{\omega^{2} \rho_{\text {avg }}^{*} \rho_{B} a^{2}(1+\nu)(1-2 \nu)}{16 \pi^{2} E_{B}} \ddot{A}\left(t^{*}\right)+ \\
\frac{E_{\text {avg }}^{*}(3-4 \nu) \pi^{2}}{2} A\left(t^{*}\right)=0 ; \\
\frac{\omega^{2} \rho_{\text {avg }}^{*} \rho_{B} a^{2}(1+\nu)(1-2 \nu)}{16 \pi^{2} E_{B}} \ddot{B}\left(t^{*}\right)+ \\
\frac{E_{\text {avg }}^{*}(3-4 \nu) \pi^{2}}{2} B\left(t^{*}\right)=0 ;
\end{gathered}
$$

$$
\begin{aligned}
& \frac{(11-18 \nu) \Delta E^{*} \pi^{2}}{27 a} C^{2}\left(t^{*}\right)-\frac{(1-\nu) E_{\mathrm{avg}}^{*} \pi^{4}}{12 \lambda} C\left(t^{*}\right)+ \\
& \frac{a(1+\nu) \Delta \theta_{0} \alpha_{B} \pi^{2}}{2}\left[E_{\mathrm{avg}}^{*} \alpha_{\mathrm{avg}}^{*} E\left(t^{*}\right)+\right. \\
& \left.\left(E_{\mathrm{avg}}^{*} \Delta \alpha^{*}+\alpha_{\mathrm{avg}}^{*} \Delta E^{*}\right) F\left(t^{*}\right)+\Delta E^{*} \Delta \alpha^{*} G\left(t^{*}\right)\right]+ \\
& \frac{q_{0} a \lambda^{2}(1+\nu)(1-2 \nu)}{E_{B}} \sin \left(2 \pi t^{*}\right)= \\
& \frac{\lambda \omega^{2} \rho_{\mathrm{avg}}^{*} \rho_{B} a^{2}(1+\nu)(1-2 \nu)}{16 \pi^{2} E_{B}} \ddot{C}\left(t^{*}\right)
\end{aligned}
$$


Table 1. Material Properties, A (Aremco Macor machinable glass ceramic), B (AISI 1010 steel).

\begin{tabular}{|c|c|c|c|c|c|}
\hline & $E(\mathrm{~Pa})$ & $\alpha\left(1 /{ }^{\circ} \mathrm{C}\right)$ & $c\left(\mathrm{~J} / \mathrm{kg}^{\circ} \mathrm{C}\right)$ & $k(\mathrm{~W} / \mathrm{mK})$ & $\rho\left(\mathrm{kg} / \mathrm{m}^{3}\right)$ \\
\hline $\mathrm{A}$ & $66.9 \times 10^{9}$ & $7.4 \times 10^{-6}$ & 790 & 1.464 & 2520 \\
$\mathrm{~B}$ & $205 \times 10^{9}$ & $12.2 \times 10^{-6}$ & 448 & 49.8 & 7870 \\
\hline
\end{tabular}

$$
\begin{aligned}
& \frac{4 \rho_{B} \rho_{\mathrm{avg}}^{*} r a^{2}}{\pi^{2} \Delta \theta_{0} k_{B}}+\frac{1}{4}\left\{-2 \pi^{2} k_{\mathrm{avg}}^{*} D(t)-2 \pi^{2} \Delta k^{*} E(t)+\right. \\
& \left.k_{\mathrm{avg}}^{*} \lambda^{2} \frac{\partial T}{\partial z^{*}}\right|_{-1 / 2} ^{1 / 2}+\left.\Delta k^{*} \lambda^{2} z^{*} \frac{\partial T}{\partial z^{*}}\right|_{-1 / 2} ^{1 / 2}- \\
& \frac{\rho_{B} c_{A} \omega a^{2}}{2 \pi k_{B}}\left[\rho_{\mathrm{avg}}^{*} c_{\mathrm{avg}}^{*} \dot{D}(t)+\left(\rho_{\mathrm{avg}}^{*} \Delta c^{*}+c_{\mathrm{avg}}^{*} \Delta \rho^{*}\right) \dot{E}(t)+\right. \\
& \left.\left.\Delta c^{*} \Delta \rho^{*} \dot{F}(t)\right]\right\}- \\
& \frac{16 E_{B} \alpha_{B} \omega}{9 \pi(1-2 \nu) k_{B}} \frac{\theta_{0}}{\Delta \theta_{0}}\left(E_{\mathrm{avg}}^{*} \alpha_{\mathrm{avg}}^{*}+\frac{\Delta E^{*} \Delta \alpha^{*}}{12}\right) C(t) \dot{C}(t)- \\
& \frac{\pi a E_{B} \alpha_{B} \omega}{4(1-2 \nu) k_{B}} \frac{1}{12 \lambda} \frac{\theta_{0}}{\Delta \theta_{0}}\left(E_{\mathrm{avg}}^{*} \Delta \alpha^{*}+\alpha_{\mathrm{avg}}^{*} \Delta E^{*}\right) \dot{C}(t)=0 ;
\end{aligned}
$$

$$
\begin{aligned}
& \frac{\rho_{B} \Delta \rho^{*} r a^{2}}{3 \pi^{2} \Delta \theta_{0} k_{B}}+\frac{1}{4}\left\{-2 \pi^{2} k_{\mathrm{avg}}^{*} E(t)-2 \pi^{2} \Delta k^{*} F(t)+\right. \\
& \left.k_{\mathrm{avg}}^{*} \lambda^{2} z^{*} \frac{\partial T}{\partial z^{*}}\right|_{-1 / 2} ^{1 / 2}-\left.k_{\mathrm{avg}}^{*} \lambda^{2} T\right|_{-1 / 2} ^{1 / 2}+ \\
& \Delta k^{*} \lambda^{2}\left(\left.z^{* 2} \frac{\partial T}{\partial z^{*}}\right|_{-1 / 2} ^{1 / 2}-\left.z^{*} T\right|_{-1 / 2} ^{1 / 2}+D(t)\right)- \\
& \frac{\rho_{B} c_{A} \omega a^{2}}{2 \pi k_{B}}\left[\rho_{\mathrm{avg}}^{*} c_{\mathrm{avg}}^{*} \dot{E}(t)+\left(\rho_{\mathrm{avg}}^{*} \Delta c^{*}+c_{\mathrm{avg}}^{*} \Delta \rho^{*}\right) \dot{F}(t)+\right. \\
& \left.\left.\Delta c^{*} \Delta \rho^{*} \dot{G}(t)\right]\right\}- \\
& \frac{4 E_{B} \alpha_{B} \omega}{27 \pi(1-2 \nu) k_{B}} \frac{\theta_{0}}{\Delta \theta_{0}}\left(E_{\mathrm{avg}}^{*} \Delta \alpha^{*}+\alpha_{\mathrm{avg}}^{*} \Delta E^{*}\right) C(t) \dot{C}(t)- \\
& \frac{\pi a E_{B} \alpha_{B} \omega}{4(1-2 \nu) k_{B}} \frac{1}{\lambda} \frac{\theta_{0}}{\Delta \theta_{0}}\left(\frac{E_{\mathrm{avg}}^{*} \alpha_{\mathrm{avg}}^{*}}{12}+\frac{\Delta E^{*} \Delta \alpha^{*}}{80}\right) \dot{C}(t)=0 ;
\end{aligned}
$$

$$
\begin{aligned}
& \frac{\rho_{B} \rho_{\mathrm{avg}}^{*} r a^{2}}{3 \pi^{2} \Delta \theta_{0} k_{B}}+\frac{1}{4}\left\{-2 \pi^{2} k_{\mathrm{avg}}^{*} F(t)-2 \pi^{2} \Delta k^{*} G(t)+\right. \\
& k_{\mathrm{avg}}^{*} \lambda^{2}\left(\left.z^{* 2} \frac{\partial T}{\partial z^{*}}\right|_{-1 / 2} ^{1 / 2}-\left.2 z^{*} T\right|_{-1 / 2} ^{1 / 2}+2 D(t)\right)+ \\
& \Delta k^{*} \lambda^{2}\left(\left.z^{* 3} \frac{\partial T}{\partial z^{*}}\right|_{-1 / 2} ^{1 / 2}-\left.2 z^{* 2} T\right|_{-1 / 2} ^{1 / 2}+4 E(t)\right)- \\
& \frac{\rho_{B} c_{A} \omega a^{2}}{2 \pi k_{B}}\left[\rho_{\mathrm{avg}}^{*} c_{\mathrm{avg}}^{*} \dot{F}(t)+\left(\rho_{\mathrm{avg}}^{*} \Delta c^{*}+c_{\mathrm{avg}}^{*} \Delta \rho^{*}\right) \dot{G}(t)+\right. \\
& \left.\left.\Delta c^{*} \Delta \rho^{*} \frac{\partial}{\partial t^{*}} \int_{-1 / 2}^{1 / 2} z^{* 4} T d z^{*}\right]\right\}- \\
& \frac{16 E_{B} \alpha_{B} \omega}{9 \pi(1-2 \nu) k_{B}} \frac{\theta_{0}}{\Delta \theta_{0}}\left(\frac{E_{\mathrm{avg}}^{*} \alpha_{\mathrm{avg}}^{*}}{12}+\frac{\Delta E^{*} \Delta \alpha^{*}}{80}\right) C(t) \dot{C}(t)- \\
& \frac{\pi a u_{0} E_{B} \alpha_{B} \omega}{320(1-2 \nu) k_{B}} \frac{1}{\lambda} \frac{\theta_{0}}{\Delta \theta_{0}}\left(E_{\mathrm{avg}}^{*} \Delta \alpha^{*}+\alpha_{\mathrm{avg}}^{*} \Delta E^{*}\right) \dot{C}(t)=0 ;
\end{aligned}
$$

$$
\begin{aligned}
& \frac{\rho_{B} \Delta \rho^{*} r a^{2}}{20 \pi^{2} \Delta \theta_{0} k_{B}}+\frac{1}{4}\left\{-2 \pi^{2} k_{\mathrm{avg}}^{*} G(t)-2 \pi^{2} \Delta k^{*} \int_{-1 / 2}^{1 / 2} z^{* 4} T d z^{*}+\right. \\
& k_{\mathrm{avg}}^{*} \lambda^{2}\left(\left.z^{* 3} \frac{\partial T}{\partial z^{*}}\right|_{-1 / 2} ^{1 / 2}-\left.3 z^{* 2} T\right|_{-1 / 2} ^{1 / 2}+6 E(t)\right)+ \\
& \Delta k^{*} \lambda^{2}\left(\left.z^{* 4} \frac{\partial T}{\partial z^{*}}\right|_{-1 / 2} ^{1 / 2}-\left.3 z^{* 3} T\right|_{-1 / 2} ^{1 / 2}+9 F(t)\right)- \\
& \frac{\rho_{B} c_{A} \omega a^{2}}{2 \pi k_{B}}\left[\rho_{\mathrm{avg}}^{*} c_{\mathrm{avg}}^{*} \dot{G}(t)+\left(\rho_{\mathrm{avg}}^{*} \Delta c^{*}+c_{\mathrm{avg}}^{*} \Delta \rho^{*}\right) .\right. \\
& \left.\left.\frac{\partial}{\partial t^{*}} \int_{-1 / 2}^{1 / 2} z^{* 4} T d z^{*}+\Delta c^{*} \Delta \rho^{*} \frac{\partial}{\partial t^{*}} \int_{-1 / 2}^{1 / 2} z^{* 5} T d z^{*}\right]\right\}- \\
& \frac{E_{B} \alpha_{B} \omega}{45 \pi(1-2 \nu) k_{B}} \frac{\theta_{0}}{\Delta \theta_{0}}\left(E_{\mathrm{avg}}^{*} \Delta \alpha^{*}+\alpha_{\mathrm{avg}}^{*} \Delta E^{*}\right) C(t) \dot{C}(t)- \\
& \frac{\pi a E_{B} \alpha_{B} \omega}{4(1-2 \nu) k_{B}} \frac{1}{\lambda} \frac{\theta_{0}}{\Delta \theta_{0}}\left(\frac{E_{\mathrm{avg}}^{*} \alpha_{\mathrm{avg}}^{*}}{80}+\frac{\Delta E^{*} \Delta \alpha^{*}}{448}\right) \dot{C}(t)=0 .
\end{aligned}
$$

Due to the fact that besides temperature integrals, temperature also appeared in the governing equations. As such, a backstepping approach was devised in the programming process. At each time step, dimensionless temperature $T$, which was only a function of $z^{*}$, was approximated by a fifth order polynomial. The coefficients of this polynomial were determined by the four temperature integrals of the preceding step and convection boundary conditions on lateral surfaces as follows:

$$
\begin{aligned}
D\left(t^{*}\right) & =\int_{-1 / 2}^{1 / 2} T\left(z^{*}, t^{*}\right) d z^{*} ; \\
E\left(t^{*}\right) & =\int_{-1 / 2}^{1 / 2} z^{*} T\left(z^{*}, t^{*}\right) d z^{*} ; \\
F\left(t^{*}\right) & =\int_{-1 / 2}^{1 / 2} z^{* 2} T\left(z^{*}, t^{*}\right) d z^{*} ; \\
G\left(t^{*}\right) & =\int_{-1 / 2}^{1 / 2} z^{* 3} T\left(z^{*}, t^{*}\right) d z^{*} ; \\
\left.k_{B} \frac{\partial \theta}{\partial z}\right|_{h / 2} & =H\left(\theta_{\infty}-\left.\theta\right|_{h / 2}\right) \\
& =H\left(\theta_{\infty}-\theta_{0}+\theta_{0}-\left.\theta\right|_{h / 2}\right) ; \\
\left.k_{A} \frac{\partial \theta}{\partial z}\right|_{-h / 2} & =-H\left(\theta_{\infty}-\left.\theta\right|_{-h / 2}\right)= \\
& =-H\left(\theta_{\infty}-\theta_{0}+\theta_{0}-\left.\theta\right|_{-h / 2}\right) .
\end{aligned}
$$

Using the temperature distribution assumption in Eq. (32) yields:

$$
\begin{aligned}
& \left.k_{B} \frac{\Delta \theta_{0}}{h} \frac{\partial T}{\partial z^{*}}\right|_{1 / 2} \sin \left(m \pi x^{*}\right) \sin \left(n \pi y^{*}\right)= \\
& H \Delta \theta_{0}-\left.H \Delta \theta_{0} T\right|_{1 / 2} \sin \left(m \pi x^{*}\right) \sin \left(n \pi y^{*}\right) \\
& \left.k_{A} \frac{\Delta \theta_{0}}{h} \frac{\partial T}{\partial z^{*}}\right|_{-1 / 2} \sin \left(m \pi x^{*}\right) \sin \left(n \pi y^{*}\right)= \\
& \quad-H \Delta \theta_{0}+\left.H \Delta \theta_{0} T\right|_{-1 / 2} \sin \left(m \pi x^{*}\right) \sin \left(n \pi y^{*}\right) .
\end{aligned}
$$

Due to the fact that the convection boundary conditions were point-wise, we satisfied them weakly by integrating over the surfaces:

$$
\begin{aligned}
\left.k_{B} \frac{\partial T}{\partial z^{*}}\right|_{1 / 2} & =\frac{\pi^{2}}{4} h H-\left.h H T\right|_{1 / 2} \\
\left.k_{A} \frac{\partial T}{\partial z^{*}}\right|_{-1 / 2} & =-\frac{\pi^{2}}{4} h H+\left.h H T\right|_{-1 / 2} .
\end{aligned}
$$




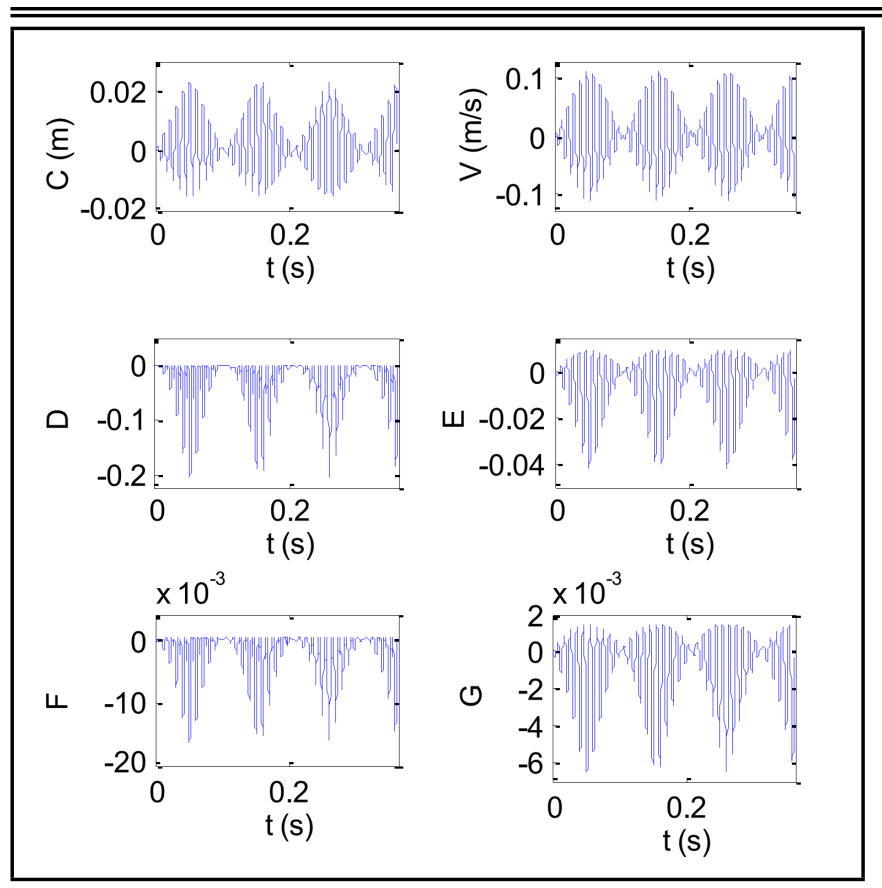

Figure 1. State variables for the base state (Lyapunov exponent = $0.2249)$.

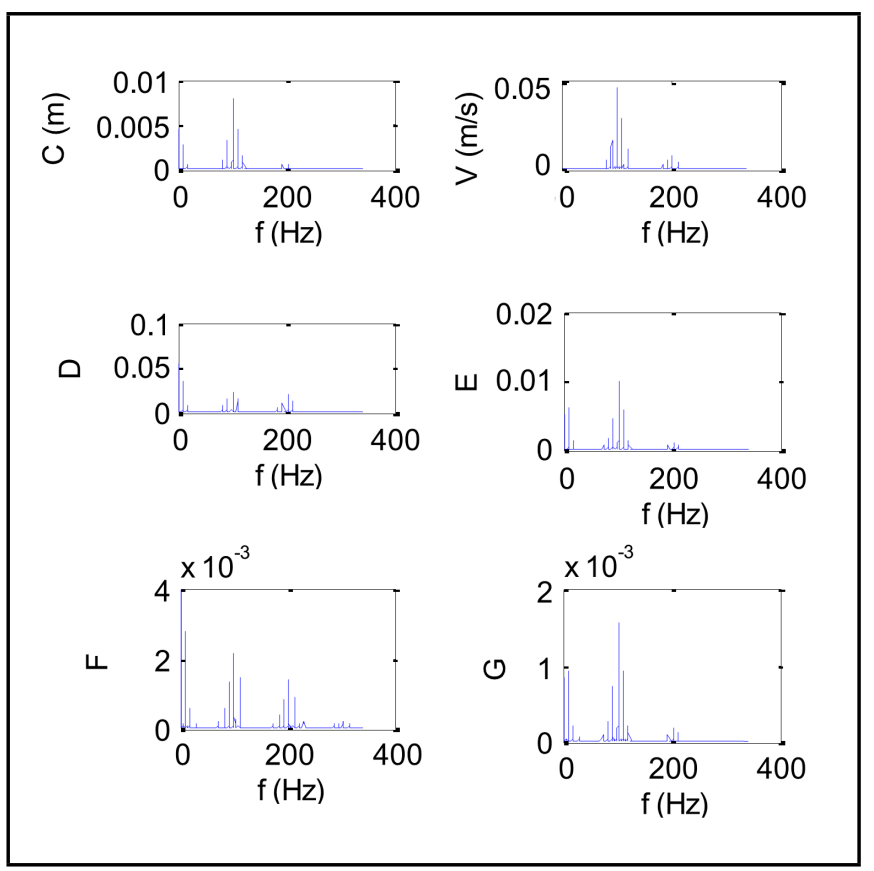

Figure 2. FFT of state variables for the base state (Lyapunov exponent $=$ $0.2249)$.

\section{RESULTS}

Equations (36) and (37), which express in-plane equations of motion, are linear and completely decoupled from parameters $C, D, E, F$, and $G$. Therefore, they can be solved separately. Solving Eqs. (38), (39), (40), (41), and (42) involves primarily changing them into six first order differential equations. Afterwards, the solution in the time domain is achieved by using the Runge-Kutta method of fourth order for an $80 \times 80 \mathrm{~cm}^{2}$ square plate with zero initial conditions for all state variables. The time step is assumed to be $1 / 50$ of the period of applied force and integration is done along 500 periods. To detect chaos, the Lyapunov exponent is calculated
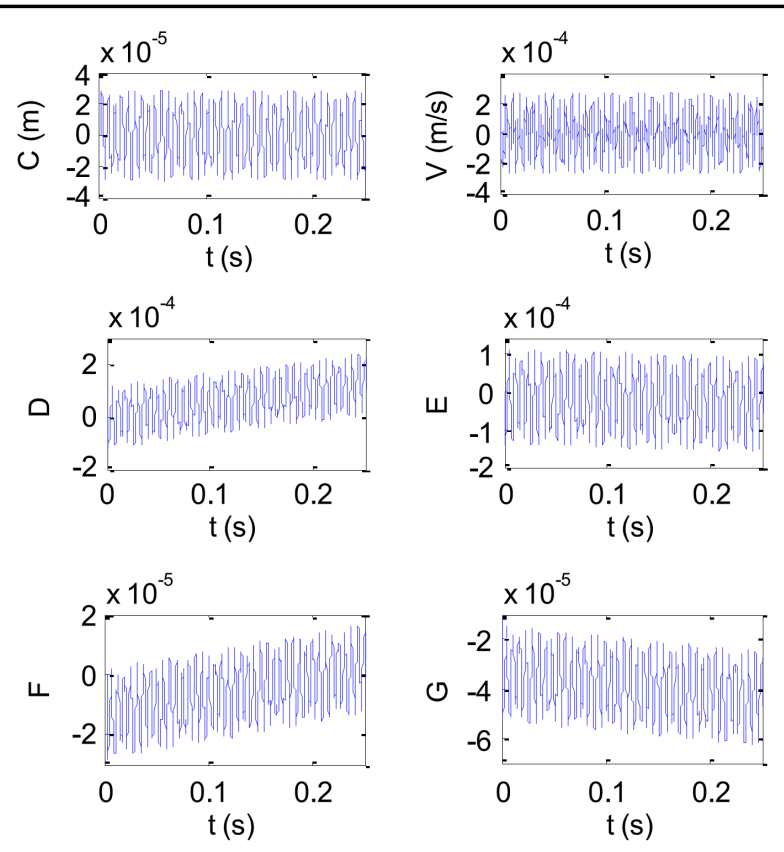

Figure 3. State variables for $\theta_{0}=290 \mathrm{~K}, q_{0}=10000 \mathrm{~N} / \mathrm{m}^{2}, r=0 \mathrm{~W} / \mathrm{kg}$, $\lambda=15$, and $\omega=1000 \mathrm{rad} / \mathrm{s}$ (Lyapunov exponent $=-0.0179$ ).

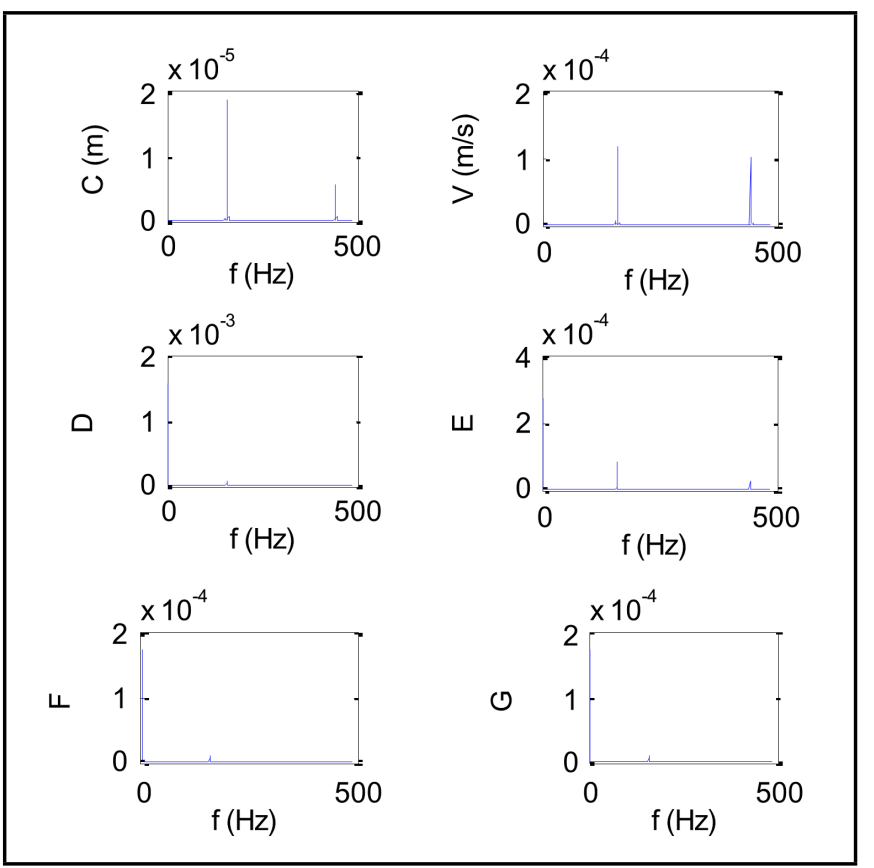

Figure 4. FFT of state variables for $\theta_{0}=290 \mathrm{~K}, q_{0}=10000 \mathrm{~N} / \mathrm{m}^{2}, r=0$ $\mathrm{W} / \mathrm{kg}, \lambda=15$, and $\omega=1000 \mathrm{rad} / \mathrm{s}$ (Lyapunov exponent $=-0.0179$ ).

for 20 initial points on each trajectory and then averaged as below: $:^{36}$

$$
\begin{gathered}
\lambda\left(x_{i}\right)=\frac{1}{n} \ln \left(\frac{d_{n}}{d_{0}}\right) ; \\
\lambda=\frac{1}{N} \sum_{i=1}^{N} \lambda\left(x_{i}\right) .
\end{gathered}
$$

If the $\operatorname{sign} \lambda$ is positive for a trajectory, it is said to be chaotic. $d_{n}$ is the evolved distance between two point of a trajectory, which were initially at the distance $d_{0}$. It should be noted that the distance $d$ indicates the Euclidean norm in six dimensional state space. Fast Fourier transform (FFT) of 
Table 2. Maximum lateral displacement, velocity, and average temperature over the thickness and Lyapunov exponent when values of heat generation, initial temperature, and lateral load change (base state: $\theta_{\text {ambient }}=300 \mathrm{~K}$, $\omega=700 \mathrm{rad} / \mathrm{s}$, and $\lambda=60 . r=0 \mathrm{~W} / \mathrm{kg}, \theta_{0}=290 \mathrm{~K}$, and $\left.q_{0}=1 \times 10^{4}\right)$.

\begin{tabular}{|c|c|c|c|c|}
\hline & $\delta_{\max }(\mathrm{m})$ & $V_{\max }(\mathrm{m} / \mathrm{s})$ & $T_{\text {avg }}(\mathrm{K})$ & LE \\
\hline Base state & 0.0233 & 0.1123 & 289.9816 & 0.2249 \\
$r=-100 \mathrm{~W} / \mathrm{kg}$ & 0.0233 & 0.1127 & 288.8077 & 0.2497 \\
$r=100 \mathrm{~W} / \mathrm{kg}$ & 0.0233 & 0.1121 & 290.2341 & 0.2190 \\
$\theta_{0}=295 \mathrm{~K}$ & 0.0233 & 0.1124 & 294.8742 & 0.1380 \\
$\theta_{0}=285 \mathrm{~K}$ & 0.0233 & 0.1123 & 285.0853 & 0.2764 \\
$q_{0}=5 \times 10^{3} \mathrm{~N} / \mathrm{m}^{2}$ & 0.0174 & 0.0912 & 290.0027 & 0.1889 \\
$q_{0}=2 \times 10^{3} \mathrm{~N} / \mathrm{m}^{2}$ & 0.0120 & 0.0669 & 290.0725 & 0.2369 \\
\hline
\end{tabular}

Table 3. Maximum lateral displacement, velocity, and average temperature over the thickness and Lyapunov exponent when frequency and side length to thickness ratio change (base state: $\theta_{\text {ambient }}=300 \mathrm{~K}, \omega=700 \mathrm{rad} / \mathrm{s}$, and $\lambda=60 . r=0 \mathrm{~W} / \mathrm{kg}, \theta_{0}=290 \mathrm{~K}$, and $\left.q_{0}=1 \times 10^{4}\right)$.

\begin{tabular}{|c|c|c|c|c|}
\hline & $\delta_{\max }(\mathrm{m})$ & $V_{\max }(\mathrm{m} / \mathrm{s})$ & $T_{\text {avg }}(\mathrm{K})$ & LE \\
\hline Base state & 0.0233 & 0.1123 & 289.9816 & 0.2249 \\
$\omega=900 \mathrm{rad} / \mathrm{s}$ & 0.0042 & 0.0222 & 290.0487 & 0.0323 \\
$\omega=1100 \mathrm{rad} / \mathrm{s}$ & 0.0021 & 0.0100 & 290.0616 & 0.0213 \\
$\omega=1300 \mathrm{rad} / \mathrm{s}$ & 0.0014 & 0.0060 & 290.0370 & -0.0128 \\
$\lambda=40$ & 0.011 & 0.0080 & 290.0632 & -0.0227 \\
$\lambda=80$ & 0.0091 & 0.0422 & 289.9619 & 0.0674 \\
$\lambda=100$ & 0.00112 & 0.0368 & 289.9171 & 0.0814 \\
\hline
\end{tabular}

the solution time series, which depicts the existing frequencies, can also be an intuitive verification to decide whether a trajectory is chaotic or not.

To investigate the conditions resulting in chaos, $\theta_{0}, q_{0}, r, \lambda$, and $\omega$ are treated as control parameters and changed in certain ranges. For different values of these parameters, the Lyapunov exponent, maximum lateral deflection, maximum lateral velocity, and average temperature over the thickness are listed in Tables 2 and 3. Figures 1, 2, 3, and 4 show the state variables and their FFT's for two cases, where the Lyapunov exponent has opposite signs. For the purpose of comparison, the problem is also solved when plate is homogeneously made of steel or ceramic. Table 4 and Fig. 5 illustrate the differences between the thermal and mechanical responses of the homogeneous plates versus the functionally graded plates.

\section{CONCLUSIONS}

In this paper, the governing equations for thermomechanically coupled nonlinear vibrations of a rectangular FGM plate are derived. Using the Galerkin approximation, the equations are changed into ordinary differential equations and solved numerically. The Lyapunov exponent is used as a criterion to recognize chaos and has been computed for various values of control parameters.

According to the results, the Lyapunov exponent is sensitive to the parameters $\theta_{0}, q_{0}, r, \lambda$, and $\omega$. However, among all the mentioned parameters, only $\lambda$ and $\omega$ can cause drastic change in the Lyapunov exponent, especially in its sign. Therefore, the geometry of trajectories in state space is strongly characterized by these two parameters and the response can be chaotic for certain values of them, as listed in Table 3 .

Table 4 and Fig. 5 show that the dynamical behavior of a functionally graded plate does not necessarily intermediate ceramic and steel plates, since many new terms emerge in the FGM governing equations through integration over the thickness due to non-homogeneity. This highlights the modeling and analysis of FGM bodies in separate contexts.
Table 4. Maximum lateral displacement, velocity, and average temperature over the thickness and Lyapunov exponent for a plate homogenously made of steel or ceramic versus FGM $\left(\theta_{\text {ambient }}=300 \mathrm{~K}, \omega=1000 \mathrm{rad} / \mathrm{s}\right.$, and $\lambda=80$. $r=0 \mathrm{~W} / \mathrm{kg}, \theta_{0}=290 \mathrm{~K}$, and $\left.q_{0}=1 \times 10^{4}\right)$.

\begin{tabular}{|c|c|c|c|}
\hline & Material A (Ceramic) & FGM & Material B (Steel) \\
\hline$V_{\max }(\mathrm{m} / \mathrm{s})$ & 0.0275 & 0.0140 & 0.0088 \\
$T_{\text {avg }}(\mathrm{K})$ & 290.1004 & 290.0365 & 290.0657 \\
$\mathrm{LE}$ & 0.0423 & -0.0664 & 0.0172 \\
\hline
\end{tabular}

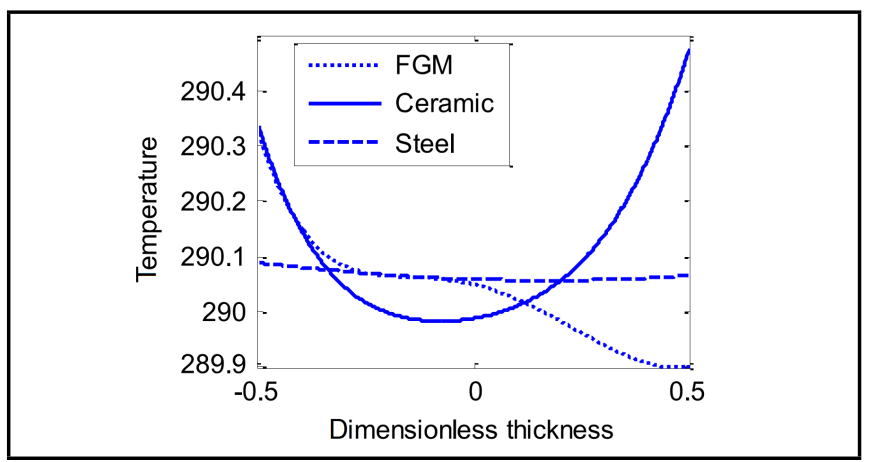

Figure 5. Temperature distribution in thickness for FGM, homogenous steel and ceramic plates $\left(\theta_{\text {ambient }}=300 \mathrm{~K}, \omega=1000 \mathrm{rad} / \mathrm{s}\right.$, and $\lambda=80 . r=$ $0 \mathrm{~W} / \mathrm{kg}, \theta_{0}=290 \mathrm{~K}$, and $\left.q_{0}=1 \times 10^{4}\right)$.

\section{REFERENCES}

1 Naghibi, S. E. and Mahzoon M. Chaotic vibrations of rectangular FGM plates with thermo-mechanical coupling, Proc. of ISME 2012 Iranian Society of Mechanical Engineers, Shiraz, Iran, (2012).

2 Nowacki, W. Dynamic Problems of Thermo-elasticity, Leyden, Noordhoff, (1975).

3 Kawamura, R., Tanigawa, Y., Kusuki, S., and Hamamura, H. Fundamental thermo-elasticity equations for thermally induced flexural vibration problems for inhomogeneous plates and thermo-elastic dynamical responses to a sinusoidally varying surface temperature, Journal of Engineering Mathematics, 61, 143-160, (2007). http://dx.doi.org/10.1007/s10665-007-9190-2

4 Xiang, W. L. and Melnik, R. Differential-algebraic approach to coupled problems of dynamic thermo-elasticity, Applied Mathematics and Mechanics, 27 (9), 1185-1196, (2006). http://dx.doi.org/10.1007/s10483-006-0905-z

5 Yang, J. and Shen, H. S. Vibration characteristics and transient response of shear deformable functionally graded plates in thermal environment, Journal of Sound and Vibration, 255 (3), 579-602, (2002). http://dx.doi.org/10.1006/jsvi.2001.4161

6 Yang, J. and Shen, H. S. Dynamic response of initially stressed functionally graded rectangular thin plates, Composite Structures, 54, 497-508, (2001). http://dx.doi.org/10.1016/s0263-8223(01)00122-2

7 Gupta, A. K. Non-linear thickness variation on the thermally-induced vibration of a rectangular plate: a spline technique, International Journal of Acoustics and Vibration, 19 (2), 131-136, (2014). http://dx.doi.org/10.20855/ijav.2014.19.2341

8 Prabhakara, M. K. and Chia, C. Y. Nonlinear flexural vibrations of orthotropic rectangular plates, Journal of Sound and Vibration, 52 (4), 511-518, (1977). http://dx.doi.org/10.1016/0022-460x(77)90367-4 
9 Allahverdizadeh, A., Naei, M. H., and Nikkhah Bahrami, M. Vibration amplitude and thermal effects on the nonlinear behavior of thin circular functionally graded plates, International Journal of Mechanical Sciences, 50, 445-454, (2008). http://dx.doi.org/10.1016/j.ijmecsci.2007.09.018

10 Allahverdizadeh, A., Naei, M. H., and Nikkhah Bahrami, M. Nonlinear free and forced vibration analysis of thin circular functionally graded plated, Journal of Sound and Vibration, 310, 966-984, (2008). http://dx.doi.org/10.1016/j.jsv.2007.08.011

11 Chang, W. P. and Chian, J. S. Nonlinear free vibration of heated orthotropic rectangular plates, International Journal of Solids and Structures, 22 (3), 267-281, (1986). http://dx.doi.org/10.1016/0020-7683(86)90091-0

12 Xuefeng, S., Xiaoqing, Z., and Jinxiang, Z. Study of nonlinear thermo-elastic free vibration of simply supported circular plate, Engineering Mechanics, 17 (2), 97-101, (2000).

13 Xuefeng, S., Xiaoqing, Z., and Jinxiang, Z. Thermo-elastic free vibration of clamped circular plate, Applied Mathematics and Mechanics, 21 (6), 715-724, (2000).

14 Praveen, G. N. and Reddy, J. N. Nonlinear transient thermoelastic analysis of functionally graded ceramic metal plates, International Journal of Solids and Structures, 35 (33), 4457-4476, (1998). http://dx.doi.org/10.1016/s00207683(97)00253-9

15 Huang, X. L. and Shen, H. S. Nonlinear vibration and dynamic response of functionally graded plates in thermal environment, International Journal of Solids and Structures, 41, 2403-2427, (2004). http://dx.doi.org/10.1016/j.ijsolstr.2003.11.012

16 Woo, J. and Meguid, S. A. Nonlinear analysis of functionally graded plates and shallow shells, International Journal of Solids and Structures, 38, 7409-7421, (2001). http://dx.doi.org/10.1016/s0020-7683(01)00048-8

17 Woo, J., Meguid, S. A., and Ong, L. S. Nonlinear free vibration behavior of functionally graded plates, Journal of Sound and Vibration, 289, 595-611, (2006). http://dx.doi.org/10.1016/j.jsv.2005.02.031

18 Chang, W. P. and Wan, S. M. Thermo-mechanically coupled nonlinear vibration of plates, International Journal of Non-Linear Mechanics, 21 (5), 375-389, (1986). http://dx.doi.org/10.1016/0020-7462(86)90021-1

19 Hao, Y. X., Zhang, W., Yang, J., and Li, S. Y. Nonlinear dynamic response of a simply supported rectangular functionally graded material plate under the timedependent thermal-mechanical loads, Journal of Mechanical Science and Technology, 25 (7), 1637-1646, (2011). http://dx.doi.org/10.1007/s12206-011-0501-1

20 Yeh, Y. L. The effect of thermo-mechanical coupling for a simply supported orthotropic rectangular plate on nonlinear dynamics, Thin Walled Structures, 43, 1277-1295, (2005). http://dx.doi.org/10.1016/j.tws.2005.03.002

21 Alijani, F. and Amabili, M. Nonlinear parametric instability of functionally graded rectangular plates in thermal environments, Proc. ASME 2012 International Mechanical Engineering Congress and Exposition, (2012). http://dx.doi.org/10.1115/imece2012-89140
22 Qiang, H., Nianmei, Z., and Guitong, Y. Chaotic motion of a nonlinear thermo-elastic elliptic plate, Applied Mathematics and Mechanics, 20 (9), 960-966, (1999). http://dx.doi.org/10.1007/bf02459058

23 Ribeiro, P. Thermally induced transition to chaos in plate vibrations, Journal of Sound and Vibration, 229, 314-330, (2007). http://dx.doi.org/10.1016/j.jsv.2006.08.003

24 Ribeiro, P. and Duarte, R. P. From periodic to chaotic oscillations in composite laminated plates, Computers \& Structures, 84, 1629-1639, (2006). http://dx.doi.org/10.1016/j.compstruc.2005.12.006

25 Lai, H. Y., Chen, C. K., and Yeh, Y. L. Double mode modeling of chaotic and bifurcation dynamics for a simply supported rectangular plate in large deflections, International Journal of Non-Linear Mechanics, 37, 331-343, (2002). http://dx.doi.org/10.1016/s0020-7462(00)00120-7

26 Yeh, Y. L., Chen, C. K., and Lai, H. Y. Chaotic and bifurcation dynamics for a simply supported rectangular plate of thermo-mechanical coupling in large deflections, Chaos, Solitons \& Fractals, 13, 1493-1500, (2002). http://dx.doi.org/10.1016/s0960-0779(01)00158-8

27 Yeh, Y. L. and Chen, C. K. Chaotic and bifurcation for a simply supported thermo-mechanical coupling circular plate with variable thickness, Chaos, Solitons \& Fractals, 22, 1013-1030, (2004). http://dx.doi.org/10.1016/j.chaos.2003.12.102

28 Zhang, W., Yang, J., and Hao, Y. Chaotic vibrations of an orthotropic FGM rectangular plate based on third order shear deformation theory, International Journal of Non-Linear Mechanics, 59, 619-660, (2010). http://dx.doi.org/10.1007/s11071-009-9568-y

29 Hao, Y. X., Chen, L. H., Zhang, W., and Lei, J. G. Nonlinear oscillations bifurcation and chaos of functionally graded materials plate, Journal of Sound and Vibration, 312, 862892, (2008). http://dx.doi.org/10.1016/j.jsv.2007.11.033

30 Sadd, M. H. Elasticity Theory, Applications, and Numerics, Academic Press, Burlington, (2009).

31 Lai, W. M., Rubin, D., and Krempl, E. Introduction to Continuum Mechanics, Butterworth Heinemann, Woburn (1999).

32 Jones, R. M. Buckling of Bars, Plates and Shells, Bull Ridge Corporation, Blacksburg Virginia, (2006).

33 Timoshenko, S. and Woinovski-Krieger, S. Theory of Plates and Shells, McGraw-Hill, Tokyo, (1984).

34 Coleman, B. D. and Noll, W. The thermodynamics of elastic materials with heat conduction and viscosity, Archive for Rational Mechanics and Analysis, 13 (1), 167-178, (1963). http://dx.doi.org/10.1007/bf01262690

35 MatWeb, LLC. MatWeb, Material Property Data. Retrieved from http://www.matweb.com, (Accessed August 14, 2014).

36 Hilborn, R. C. Chaos and Nonlinear Dynamics, Oxford University Press, New York, (2000). 